\title{
Travel Time and Point Speed Fusion based on a Macroscopic Traffic Model and Non-linear Filtering
}

\author{
David Gundlegård, Andreas Allström, Erik \\ Bergfeldt, Rasmus Ringdahl \\ Department of Science and Technology \\ Linköping University \\ Norrköping, Sweden
}

\author{
Alexandre M. Bayen \\ Department of Civil and Environmental Engineering \\ University of California, Berkeley \\ Berkeley, USA
}

\begin{abstract}
The number and heterogeneity of traffic sensors are steadily increasing. A large part of the emerging sensors are measuring point speeds or travel times and in order to make efficient use of this data, it is important to develop methods and frameworks for fusion of point speed and travel time measurements in real-time. The proposed method combines a macroscopic traffic model and a non-linear filter with a new measurement model for fusion of travel time observations in a system that uses the velocity of cells in the network as state vector. The method aims to improve the fusion efficiency, especially when travel time observations are relatively long compared to the spatial resolution of the estimation framework. The method is implemented using the Cell Transmission Model for velocity (CTM-v) and the Ensemble Kalman Filter (EnKF) and evaluated with promising results in a test site in Stockholm, Sweden, using point speed observations from radar and travel time observations from taxis.
\end{abstract}

Keywords - Data fusion, Ensemble Kalman Filtering, Cell Transmisson Model, traffic state estimation.

\section{INTRODUCTION}

Traffic management centers are facing a large and increasing number of different types of traffic sensors. The number of sensors available from crowdsourcing and other fleets that can share traffic data based on new types of business models for traffic sensing is increasing dramatically. At the same time dedicated traffic sensors are getting cheaper, less intrusive and more accurate. However, no single sensor or data source will be sufficient to enable efficient traffic monitoring in a city-wide level. Instead, current and future traffic management centers will have to rely on a large number of heterogeneous sensors to provide accurate, robust, timely and cost efficient road traffic state estimation and prediction, and this requires efficient methods for fusing the data from the different types of sensors.

A large part of the emerging sensors are measuring point speeds or travel times instead of occupancy or flow, which traditionally has been the most common observations. This makes it important to develop traffic models and frameworks for data fusion that can handle these type of measurements efficiently. Point speeds typically describe the traffic state in a specific point in the network well, whereas travel times capture in a good way an aggregate of the traffic state for the spatial extension of the measurement. This makes it very appealing to combine these two measurements to get a good base for network-wide traffic state estimation.
The aim of this paper is to use a macroscopic traffic model together with a non-linear filtering framework for efficient fusion of point speeds and travel times. The remainder of the paper is structured as follows. Section II describes different types of sensors that provide point speeds and travel times as well as some important characteristics related to sampling. Section III defines the estimation problem that we are focusing on and section IV describes previous work on the topic. Section $\mathrm{V}$ describes the data assimilation and fusion framework in more detail. Section VI describes the observation models used for fusion of the different observations. Section VII includes results from a highway test site in Stockholm whereas Section VIII concludes the paper with discussion and conclusions.

\section{BACKGROUND}

The aim of systems for traffic state estimation is to estimate the evolution of the traffic state in space and time. For practical reasons we are not able to observe state variables continuously everywhere, which means that we have to sample our observations according to some strategy. Furthermore, the number of observations, the sensing infrastructure and the traffic model are limiting the possible resolution of the system in space and time, which means that we have to work with aggregates of the observations in these dimensions.

The sampling and aggregation strategy are quite important for the performance of the estimation and are also varying between different types of sensors. This needs to be accounted for in an efficient fusion framework. Eulerian sensors, such as fixed radar stations or license plate cameras, generate observations in specific points in space. These observations are either point observations that are aggregated locally, e.g. point speeds, or contain some kind of identity that can be matched in downstream sensors and then spatially aggregated, e.g. travel times.

Lagrangian sensors are located in vehicles and observations are made while moving with the traffic, typical examples are different types of devices, e.g. navigation or fleet management equipment, reporting GPS measurements such as location and local speed. In many cases also an identity of the device is included, which means that travel time observations can be made. Lagrangian sensors are more flexible in sampling strategy and are not bounded to space-based sampling, i.e. 
observations triggered by passing a specific location, instead time-based sampling, i.e. observations triggered by a time period, is often used.

Both Eulerian and Lagrangian sensors can use point measurements or spatial aggregations where the observations are aggregated in space. Spatial aggregation is affecting both the spatial and temporal resolution, since it takes time to traverse the spatial extension of the observation.

Aggregation of measurements are always made in different levels in systems for data collection. In traffic state estimation, aggregations are typically performed in both time and space and the level of aggregation affects the delay of the system as well as the possible spatiotemporal resolution of the system. Travel time observations are interesting since they incorporate an inherent spatiotemporal aggregation, i.e. the observation is a spatial aggregate of vehicle speed during the time for traversing the given space. Both the spatial and temporal aggregation of travel time observations need attention when fusing it with other sensors, especially when it is used in real-time applications, since the maximum inherent delay of the observation is equal to the travel time itself.

The spatial aggregation distance for most of the currently used travel time sampling periods is in many cases longer than the spatial resolution of the system, i.e. simply normalizing the travel time observation with traversed distance to generate a section speed observation does not capture spatial speed variations efficiently. Figure 1 shows the test site used in this paper including sensors; Bluetooth and Radar (Eulerian) to the left and GPS probes (Lagrangian) to the right. Figure 2 shows the southbound GPS probes in Figure 1 after map matching and the grid shows the spatiotemporal resolution of 200 meters by 60 seconds. It can be seen that, unless we are in severe congestion, a time-based sampling of 60-180 seconds generate spatial aggregation distances up to a factor of 10 or more compared to the system resolution, which motivates work on allocating the observation in space more efficiently.

The type of spatial travel time allocation method will be most important when the observation contains large speed variation, which is most likely to occur when the traversed distance is long, i.e. for space-based or time-based sampling with low resolution. However, for time-based sampling the problem is reduced for low speeds whereas for space-based it does not depend on the average speed.

Furthermore, if the travel time is longer than the temporal resolution of the estimation framework, the observation will not only reflect the system at the current state, but also previous time steps. If a time-based sampling is used that has a sampling time close to or smaller than the temporal resolution of the system, this does not cause any major problems. This is typically the case for sensors like GPS-probes, where the location sampling is in the order of 1 minute or less. If, on the other hand, space-based sampling is used for the travel times, the aggregation period is increasing with reduced speed, which can cause very large delays for parts of the observations, depending on the distance between observations. This is typically the case for sensors like licence plate cameras or Bluetooth readers.

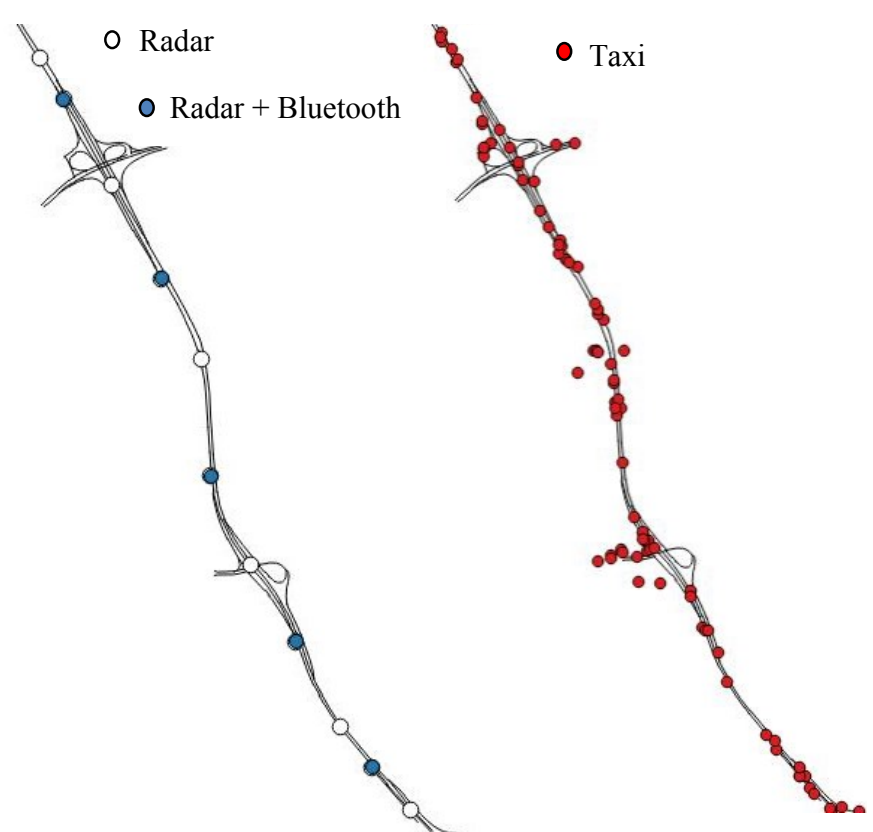

Figure 1. The test site with sensors used for experiments in this paper. To the left placement of the Eulerian Bluetooth and radar sensors. To the right example of Lagrangian GPS probe sensor observations from Taxis.

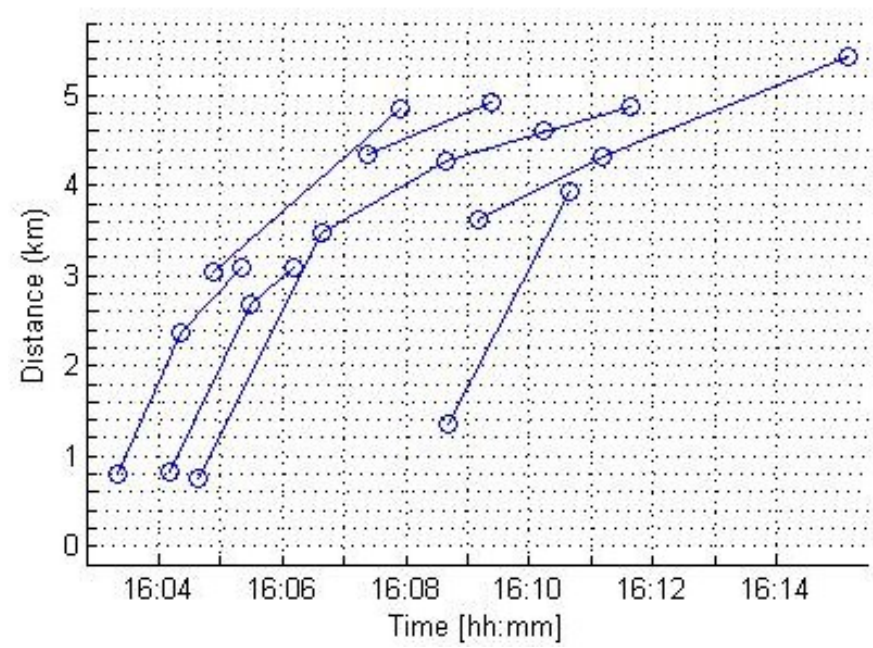

Figure 2. Southbound space-time trajectories of the GPS probe observations shown in Figure 1 after map matching. The grid indicates the spatiotemporal resolution of the state estimation system. The GPS probes are taxis in Stockholm with sample time varying between 60 and 180 seconds.

Table 1 summarizes the most commonly used traffic sensors today together with the type of observation the sensor produces as well as the sampling strategy. It can be seen that many sensors observe space-based travel times. However, it should also be noted that Loop detectors and Radar are extensively 
deployed today and that the use of time-based GPS probes are increasing dramatically.

Table 1. Overview of the most commonly used traffic sensors today and the sampling strategy that is used.

\begin{tabular}{lll}
\hline Sensor & Observation & Sampling type \\
\hline Radar & Speed and flow & Space-based \\
Loop detector & Occupancy & Space-based \\
GPS probe & Speed and/or Travel time & Time-based \\
Bluetooth & Travel time & Space-based \\
LPR camera & Travel time & Space-based \\
Cellular probe & Travel time & Space-based \\
$\begin{array}{l}\text { Loop detector with re- } \\
\text { identification }\end{array}$ & Travel time & Space-based \\
Toll tag reader & Travel time & Space-based \\
\hline
\end{tabular}

\section{PROBLEM FORMULATION}

The aim of the system is to perform traffic state estimation on a given road network. The state variable to estimate is typically speed, density or flow, depending on the intended application. These variables are all connected through the hydrodynamic relation and the fundamental diagram.

We have chosen to use the speed as state variable and hence the aim is to estimate how the average speed propagates in space and time based on the observations we have available from different types of sensors. In order to improve the estimation, we also use a first order traffic flow model for the state propagation, which can be used for assimilation of model predictions and sensor observations.

The state space model is formulated according to:

$$
\begin{aligned}
& v^{n}=M\left(v^{n-1}\right)+\eta^{n} \\
& y_{k}^{n}=h_{k}\left(v^{n}\right)+\chi_{k}^{n} \\
& \eta^{n} \propto\left(\mu_{\mathrm{mod}}, Q^{n}\right) \\
& \chi_{k}^{n} \propto\left(\mu_{o b s}, R_{k}^{n}\right)
\end{aligned}
$$

where $v^{n}$ is the state vector in time step $n$, including the speed for each part of the road network (cell) according to the spatial resolution of the system. $M(\cdot)$ is the system model, in this paper a Macroscopic traffic model. $y^{n}$ is the observation vector in time step $n$ and $h_{k}($ ) is the observation model for observation type $k$. $\eta$ and $\chi$ is the possibly time-varying model and observation noise, respectively. Given a specific fusion framework and a state vector, the EnKF and cell speeds in our case, the aim is to find $h_{k}($ ) for the travel time observation to enable an efficient update of cell speeds given the observation.
The problem is similar to the link travel time decomposition problem, where the aim is to assign contributions of a route travel time, typically as observed by sparsely sampled probes, to the set of links included in the route. Both problems are underdetermined, since many combinations of cell speeds or link travel times can correspond to a specific route travel time.

A straightforward and naïve way of solving the problem is to calculate the average speed of the travel time and use that average speed as observations on all cells traversed in the observation. However, this approach does not take into consideration the speed variation in space during the observation. A corresponding naïve solution to the link travel time decomposition problem is to allocate the travel time of links according to the distance traversed in each link.

A more sound method aims at estimating the most likely speed profile for the travel time observation, given some external input and use that speed profile as input to the traffic estimation. The external input can be information about the road network, traffic models and other observations. In this paper we use a first order traffic model, point speed measurements and other travel time measurements together with a non-linear filtering technique to estimate the most likely speed profile.

\section{PREVIOUS WORK}

Data fusion is a well-established research topic within the military and aircraft sector, see for example [1] and [2]. Within the transport sector the interest for data fusion has, for natural reasons, increased as new types of data have been made available. The rapid increase of new data sources during the last decade has also made the task more complex and consequently further research is required.

In a survey on data fusion methods in Intelligent Transportation Systems (ITS) El Fazoui et al. [3] concluded that there are several methods available that can be used to fuse heterogeneous data. The methods range from more naïve statistical approaches as different weighting methods to more complex methods as neural networks and Kalman filters. However, the conclusion made by El Fazoui et al. was that none of the studied methods used for traffic estimation produce accurate estimations and predictions except for some special conditions. In order to achieve accurate results for a variety of network configurations and data sets available a combination of different approaches is recommended. One proposed solution is to assimilate estimations of the same quantity from different prediction methods. It is noted that various Kalman filters and particle filters are well established methods used for data assimilation.

Byon et al. [4] used a Single-Constraint-At-A-Time (SCAAT) Kalman filter that uses the single most recent speed measurement from any available sensor, in this case loop detectors and GPS probe data. The state is updated based on the characteristics of that particular sensor (i.e. the noise and variance of the measurement) and the accumulated state 
estimation from the previous step. The results are promising but the limitation is that only speed measurements can be used.

Bachmann et al. [5] [6] compared different methods for fusing speed from loop detector data with travel times collected with Bluetooth. This was done both on collected data and simulated data. Among the evaluated methods was neural network, measurement fusion Kalman filter, SCAAT Kalman filter, fuzzy integral, ordered weighted averaging and a simple convex combination. The results show that most data fusion techniques improve the accuracy over single sensor approaches. The measurement fusion Kalman filter and the simple convex combination perform well in all scenarios and often significantly improve the accuracy of the estimations. The authors also conclude that the measurement fusion Kalman filter would be particularly useful if flow and density also would be available. However, this implementation of the Kalman filter requires measurement from all sensors to produce a fused estimate. The measurement fusion Kalman Filter is a multisensor multi-temporal Kalman filter presented by Mitchell [7].

Treiber and Helbing [8] introduced the Adaptive smoothing method, which is a nonlinear spatiotemporal lowpass filter that uses the information from loop detectors to reconstruct the spatiotemporal traffic dynamics for a certain road network. The proposed method is based on a number of parameters and some of them are traffic flow related such as the propagation velocity in congestion and free-flow. In accordance with the kinematic wave theory the method assumes that the information travels in the direction of traffic flow when in free-flow, while the information travels against the direction of traffic while in congestion. Each data point is assigned a weight which is based on the spatiotemporal distance from the point where the traffic state is estimated. This method was later extended and generalized by van Lindt and Hoogendoorn [9] to also handle other types of data. The so-called Extended Generalized Treiber-Helbing filter (EGTF) can handle all kinds of data, no matter their spatial and temporal resolution, as long as the data provides a mean to distinguish between free flow and congestion. The original filter proposed by Treiber and Helbing assumed that the data was collected at fixed points and at fixed time interval. However, in the EGTF the data can stem from any position and time. Two weights are introduced where the first one is a data specific weight which can be interpreted as the reliability of that particular data source. The second weight is data source and data point dependent and reflect that fact that data from different sources will result in different distinctions between free-flow and congestion. The EGFT is evaluated both on simulated data [9] and on empirical data [10] with promising results. In [10] the method is compared with piecewise linear and quadratic speed-based interpolation methods and as earlier concluded by van Hinsbergen et al. [11] using kinematic wave theory in combination with filtering technique result in more accurate travel time estimations in comparison to different interpolation methods.
Work et al. [12] proposed a modified version of the Cell Transmission Model named the Cell Transmission Model for velocities (CTM-v) that together with an ensemble Kalman filter (EnKF) was used to fuse speed measurements from loop detectors and probe data. This method was developed within the Mobile Millennium project to run in real-time and estimate the traffic state for the highway network around San Francisco. Mazare et al. [13] evaluated almost 1000 scenarios where the CTM-v and EnKFr was used to fuse probe and loop detector data and estimate travel times. The results clearly showed that when complementing loop detector data with probe vehicle data, better estimates for travel times were obtained. However, this implementation of the CTM-v and the EnKF can only handle point speed measurements, not travel times.

Regarding the link travel time decomposition problem Hofleitner and Bayen [14] proposed a method for arterials based on a probability distribution of the travel time. The probability is based on the traffic dynamics of the specific links and the hydrodynamic theory. The method is evaluated using a traffic simulation tool and the results show that it provides a significant improvement compared to a benchmark method that allocates the travel time proportionally to the free flow speed on each link.

Qing et al. [15] proposed a method called TravRes that can be used to reconstruct the shape of trajectories. TravRes uses an iterative approach and is based on a method called PISCIT. The TravRes method assumes that each cell in the space-time diagram has homogenous traffic conditions and that the floating car data are strictly consistent and can be exactly reconstructed from the time-space speeds. TravRes consist of an inner and an outer iteration loop where the inner loop reconstructs the trajectories and the outer loop reconstructs the time-space speeds. The two loops are iteratively ran until the given floating car data are consistent enough with the time-space speeds. The validation of the method shows that only a few iterations often are enough to improve the estimation error and the inconsistency. The TravRes method can also be used to fuse floating car data with local speed measurements or travel times.

\section{SYSTEM DESCRIPTION}

The operator $M(\cdot)$ in the state space model (1) is a macroscopic traffic model, the cell transmission model for velocity (CTM-v) in our case. The CTM-v model [12] is a first order model based on the Lighthill-Whitham-Richards (LWR) partial differential equation (PDE) [16] discretized using the Godunov scheme [17] and then translated from density to velocity using the hyperbolic-linear fundamental diagram [12]. Due to the nonlinearity and non-differentiability of the model and a relatively large state-vector, the method chosen for data assimilation and fusion is the ensemble Kalman filter. The EnKF and CTM-v framework is first described in [12] where details about the model as well as the filtering can be found. The adaption of the framework for the Stockholm network, which is used in this paper, is described in [18]. 
Given the forecasted $\left(P_{f}^{n}\right)$ and the analyzed $\left(P_{a}^{n}\right)$ estimate of the state covariance in time step $n$, the EnKF algorithm can be summarized as follows [12][19]:

1. Initialization: Draw $K$ ensemble realizations $v_{a}^{0}(k)$ $k \in\{1, \ldots, K\}$ from a process with mean speed $v_{a}^{0}$ and covariance $P_{a}^{0}$.

2. Forecast (time update): Update each of the $K$ ensemble members according to the CTM-v algorithm [12], then update the ensemble mean and covariance according to:

$$
\begin{gathered}
v_{f}^{n}(k)=M\left(v_{a}^{n-1}\right)+\eta^{n}(k) \\
\bar{v}_{f}^{n}(k)=\frac{1}{K} \sum_{k=1}^{K} v_{f}^{n}(k) \\
P_{f}^{n}=\frac{1}{K-1} \sum_{k=1}^{K}\left(v_{f}^{n}(k)-\bar{v}_{f}^{n}\right)\left(v_{f}^{n}(k)-\bar{v}_{f}^{n}\right)^{T}
\end{gathered}
$$

3. Analysis (measurement update):

$$
\begin{gathered}
G^{n}=P_{f}^{n}\left(H^{n}\right)^{T}\left(H^{n} P^{n}\left(H^{n}\right)^{T}+R^{n}\right)^{-1} \\
v_{a}^{n}(k)=v_{f}^{n}(k)+G^{n}\left(y^{n}-H^{n} v_{f}^{n}(k)+\chi^{n}(k)\right) \\
P_{f}^{n}=\frac{1}{K-1} \sum_{k=1}^{K}\left(v_{f}^{n}(k)-\bar{v}_{f}^{n}\right)\left(v_{f}^{n}(k)-\bar{v}_{f}^{n}\right)^{T}
\end{gathered}
$$

\section{Return to 2 .}

In the above description, $H^{n}$ refers to the linear observation model, $G^{n}$ is the Kalman gain and $y^{n}$ is the observation in time step $n$.

\section{OBSERVATION MODELS}

To enable efficient fusion of observations with different modality, we need to define suitable observation models, which are further described in this section. The model for point speed observation update is defined as in [12]:

$$
y_{p}^{n}=H_{p}^{n} v^{n}+\chi_{p}^{n}
$$

where $H_{p}^{n} \in\{0,1\}^{p^{n} \times K}$

$p^{n}=$ discrete cells for which velocity measurements from radar are observed during time step $n$

$K=$ total number of cells in the network

The point speed observation update is made in a batch for all observations in a time step. However, the travel time observations are assumed independent and updated in sequence:

$$
y_{t_{i}}^{n}=H_{t_{i}}^{n} v^{n}+\chi_{t_{i}}^{n}
$$

where $t_{i}$ is the travel time measurement $i$ in time step $n$. For the filter to be able to handle the measurements efficiently, we need to express the travel time measurement as a function of the state, i.e. we need to define the observation model that maps the travel time measurements to our state variables, which is the average speed in the different cells. There is a simple relationship between average speed and travel time:

$$
t=\frac{S}{\bar{v}}
$$

where $t$ is the travel time, $S$ is the distance traveled and $\bar{v}$ is the average speed. If the speed varies over the stretch $S$, the travel time $t$ as a function of the state $v$ becomes

$$
t(v)=\int_{0}^{S} \frac{1}{v(s)} d s
$$

In practice, we need to discretize our system, hence, with a set of discrete cells with length $s_{i}$, the travel time becomes

$$
t=\sum_{j \in J} \frac{s_{j}}{v_{j}}
$$

where $v_{j}$ is the average speed in cell $j$ and $J$ is the set of cells traversed in the travel time observation. The travel time observation is a nonlinear function of the state, which means that we can not use this observation model directly in the filter. We can handle this in two ways, 1) linearizing the observation model or 2) estimating the observation model by an ensemble of realizations from a priori model probability density function. First, we try to linearize the measurement model.

Let us linearize the measurement equation using a first order Taylor approximation in the point $\hat{v}$ :

$$
L_{t}(\hat{v})=t(\hat{v})+\frac{\partial t(\hat{v})}{\partial \hat{v}}(v-\hat{v})=\sum_{i \in J}\left(\frac{2 s_{j}}{\hat{v}_{j}}-v_{j} \frac{s_{j}}{\hat{v}_{j}^{2}}\right)
$$

where $\hat{v}_{j}$ is the most recent estimate of the state in cell $j$.

We measure travel time number $i$ in time step $n$ and let us call that $\Delta t_{i}^{n}$. To be able to express the measurement model in matrix form, we modify our observation of travel time $i$ in time step $n$ to:

$$
y_{t_{i}}^{n}=\sum_{j \in J} \frac{2 s_{j}}{\hat{v}_{j}^{n}}-\Delta t_{i}^{n}
$$

where $s_{j}$ is the traversed distance in cell $j$. Now, observation $i$ in time step $n$ can be expressed as 


$$
\begin{aligned}
& y_{t_{i}}^{n}=\sum_{j \in J} \frac{2 s_{j}}{\hat{v}_{j}}-\Delta t_{i}^{n}= \\
& \sum_{j \in J} \frac{2 s_{j}}{\hat{v}_{j}}-\sum_{j \in J}\left(\frac{2 s_{j}}{\hat{v}_{j}^{n}}-v_{j}^{n} \frac{s_{j}}{\left(\hat{v}_{j}^{n}\right)^{2}}\right)=\sigma_{i} \phi_{i} v^{n}
\end{aligned}
$$

where $\sigma_{i}$ is a $1 \times \mathrm{K}$ vector with the traveled distance in each cell for observation $i$ and $\phi_{i}$ is a $1 \times K$ vector with each element being $1 /\left(\hat{v}_{j}^{n}\right)^{2}$ multiplied with a $K x K$ unity matrix. $\sigma_{i} \phi_{i}$ is now the travel time observation matrix previously denoted $H_{t_{i}}^{n}$.

For the linearization approach, the analysis step (5)-(7) of the EnKF algorithm is now done once for all point observations and then repeated for all travel time observations with $H^{n}$ in (5) and (6) replaced with $H_{p}$ and $H_{t}$ for the point observation and travel time observation update, respectively.

The second approach to handle the nonlinear observation model is to use the same ensemble approximation that is used for the CTM-v model. This could reduce the effect of linearization errors that occur if the estimated state vector after the point speed observation update differs significantly from the true state. In this approach, (5) and (6) in the travel time observation update step are replaced with:

$$
\begin{gathered}
G^{n}(k)=P_{f}^{n}\left(H^{n}(k)\right)^{T}\left(H^{n}(k) P^{n}\left(H^{n}(k)\right)^{T}+R^{n}\right)^{-1} \\
v_{a}^{n}(k)=v_{f}^{n}(k)+G^{n}(k)\left(y^{n}-H^{n}(k) v_{f}^{n}(k)+\chi^{n}(k)\right)
\end{gathered}
$$

Note that the Kalman gain $(G)$ and the observation matrix $(H)$ have to be updated for each ensemble realization.

\section{RESULTS}

The assimilation and fusion framework presented in this paper can be used for many different combinations of traffic sensors. Two very interesting scenarios are where we want to use e.g. taxi probes to improve estimation performance in roads that are poorly equipped with point speed sensors or if we want to utilize both point speed and travel time observations where we have probes that can report both point speed and an identity that is consistent between at least two observations. We have evaluated the framework in the Stockholm test site (Figure 1) where radars are used for point speed observations, taxi probes are reporting time-based travel times and Bluetooth detectors are used for space-based travel times. Four scenarios, as described in Table 2, are evaluated in this paper. The scenarios are evaluated against Bluetooth observations in the case of travel times and against a full set of radar sensors (6 sensors) for space-time-speed diagrams. The model parameters (sources, sinks and split ratios) are calibrated against incident-free days of February 2013 and evaluation is made for three incident-free days in March 2013. The EnKF parameters for the different measurements are calibrated for one incident-free day in March using the framework described in [20]. The results of the calibration, the model noise mean and standard deviation $\left(\mu_{\text {mod }}, Q\right)$, the radar observation noise mean and standard deviation $\left(\mu_{\text {radar }}, R_{\text {radar }}\right)$ and the taxi observation noise mean and

\begin{tabular}{|c|c|}
\hline Scenario & Description \\
\hline 1 & $\begin{array}{l}\text { CTM-v model only. Sources and sinks predicted } \\
\text { using historic observations. }\end{array}$ \\
\hline 2 & CTM-v model and taxi observations. \\
\hline 3 & $\begin{array}{l}\text { CTM-v model and two radar observations in start and } \\
\text { end of test site. }\end{array}$ \\
\hline 4 & $\begin{array}{l}\text { CTM-v model, two radar observations and taxi } \\
\text { observations. }\end{array}$ \\
\hline 5 & CTM-v model and all radar observations. \\
\hline 6 & $\begin{array}{l}\text { CTM-v model, all radar observations and taxi } \\
\text { observations. }\end{array}$ \\
\hline
\end{tabular}
standard deviation $\left(\mu_{\text {taxi }}, R_{\text {taxi }}\right)$, is shown in Table 3.

Table 2. Scenarios for evaluation against Bluetooth observations and a full set of radar sensors (six sensors).

Table 3. EnKF parameter values from the calibration.

\begin{tabular}{cccc}
\hline Scenario & $\left(\mu_{\mathrm{mod}}, Q\right)$ & $\left(\mu_{\text {radar }}, R_{\text {radar }}\right)$ & $\left(\mu_{\text {taxi }}, R_{\text {taxi }}\right)$ \\
\hline 2 & $(0.003,0.551)$ & - & $(13.950,43.795)$ \\
3 & $(0.005,0.881)$ & $(1.016,2.503)$ & - \\
4 & $(0.005,0.881)$ & $(0.173,0.883)$ & $(18.197,47.230)$ \\
5 & $(0101,2.365)$ & $(1.165,1.294)$ & - \\
6 & $(0.036,1.853)$ & $(0.667,0.113)$ & $(44.541,47.234)$ \\
\hline
\end{tabular}

Figure 3 shows the travel time for a part of the $3 \mathrm{~km}$ long southbound stretch in Figure 1 using different combinations of sensors in the assimilation and fusion framework. The Bluetooth travel time estimate is assumed to be a good benchmark for the travel time estimates based on radar and taxi measurements. Table 4 shows the Mean Average Percentage Error (MAPE) of the different model and sensor combinations using Bluetooth as reference. The travel time MAPE is defined as:

$$
M A P E_{t t}=\frac{1}{N} \sum_{j \in J}\left|\frac{t t_{j}^{e s t}-t t_{j}^{r e f}}{t t_{j}^{r e f}}\right|
$$

Table 4 indicates significant improvements for all scenarios when combining the point speed observations from radar with the travel time observations from taxis compared to the Bluetooth observations. In Figure 3 it can be seen that although the model together with the two radar observations performs well during the peaks due to the similarity with a regular day, 
the fusion with travel times increases the accuracy especially during build-up and discharge of the congestion.

Table 4. Travel time MAPE values for different sensor combinations

\begin{tabular}{ccccc}
\hline Scenario & March 19 & March 20 & March 21 & March 22 \\
\hline 1 & 0.701 & 0.583 & 0.262 & 0.667 \\
2 & 0.233 & 0.228 & 0.213 & 0.263 \\
3 & 0.134 & 0.154 & 0.178 & 0.151 \\
4 & 0.119 & 0.126 & 0.154 & 0.126 \\
5 & 0.049 & 0.067 & 0.058 & 0.053 \\
6 & 0.049 & 0.052 & 0.055 & 0.047 \\
\hline
\end{tabular}

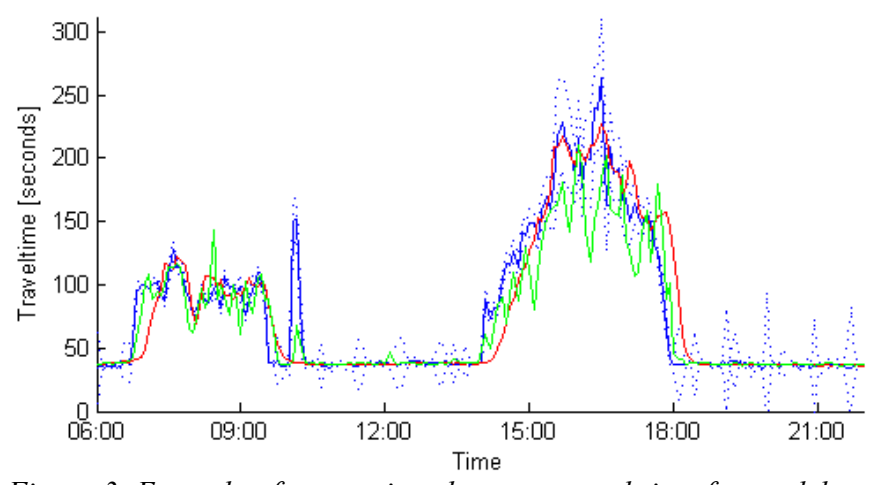

Figure 3. Example of comparison between travel time for model and two radar sensors (red), model, two radar sensors and taxi (green), Bluetooth (blue) and Bluetooth 95\% confidence interval (dotted blue).

Since the aim of the system is to estimate how the velocity field propagates in time and space we also evaluate the framework using space-time-speed plots. Examples of space-time-speed plots for the different scenarios are shown in Figure 4 to Figure 8. From the figures it can be seen that there is a large improvement to add the taxi data compared to only use the model. Furthermore, it can be noted that the start and end of the congestion seems to be more accurate in time when combining the taxi data with the radar data, compared to just using radar data.

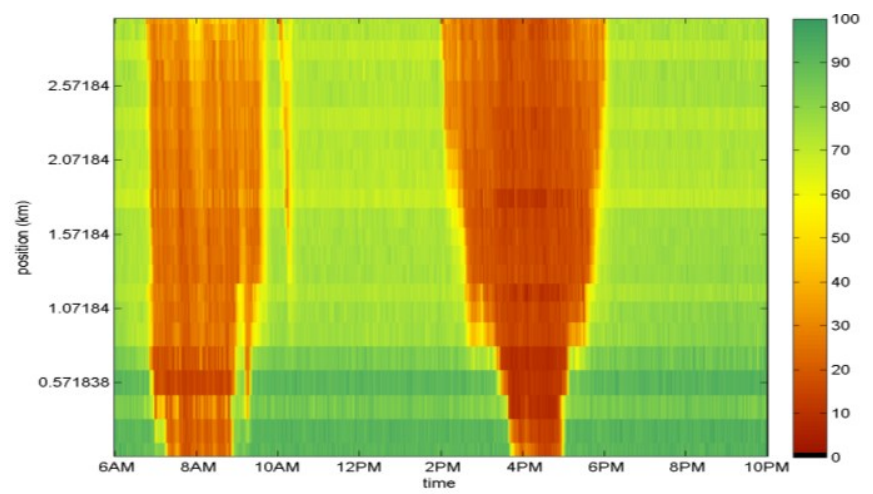

Figure 4. Space-time-speed plot for model and all radar sensors, this is the benchmarking setting for the space-time evaluations. The colorbar shows the speed in $\mathrm{km} / \mathrm{h}$.

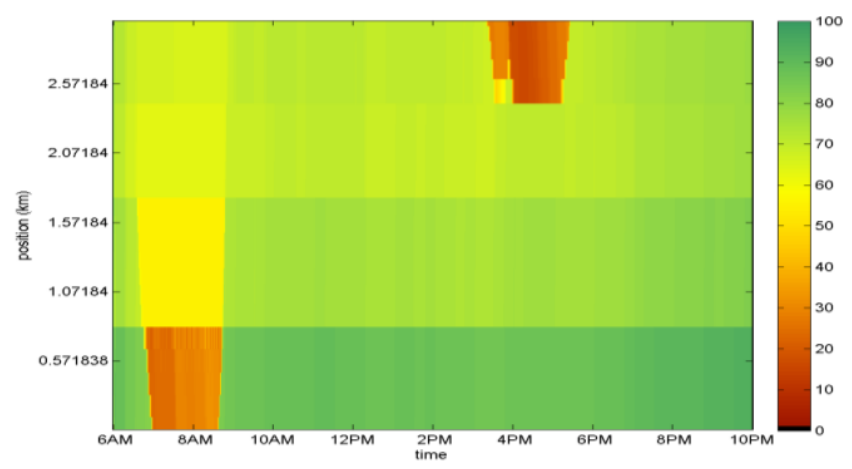

Figure 5. Space-time-speed plot for the model only (Scenario 1). The color-bar shows the speed in $\mathrm{km} / \mathrm{h}$.

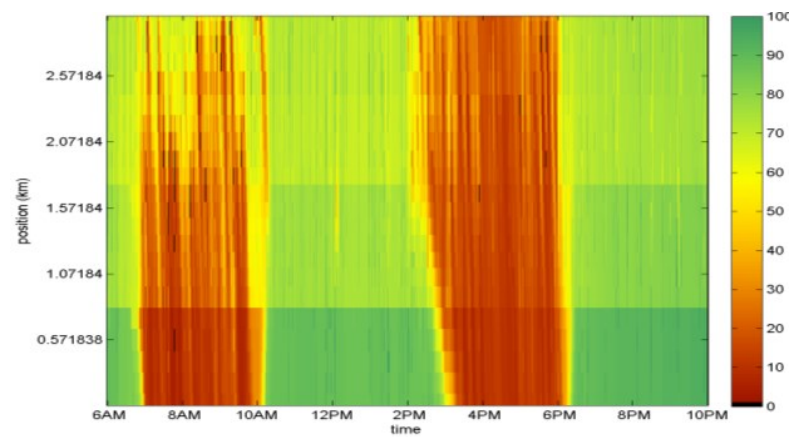

Figure 6. Space-time-speed plot for the model and taxi sensors (Scenario 2). The color-bar shows the speed in $\mathrm{km} / \mathrm{h}$.

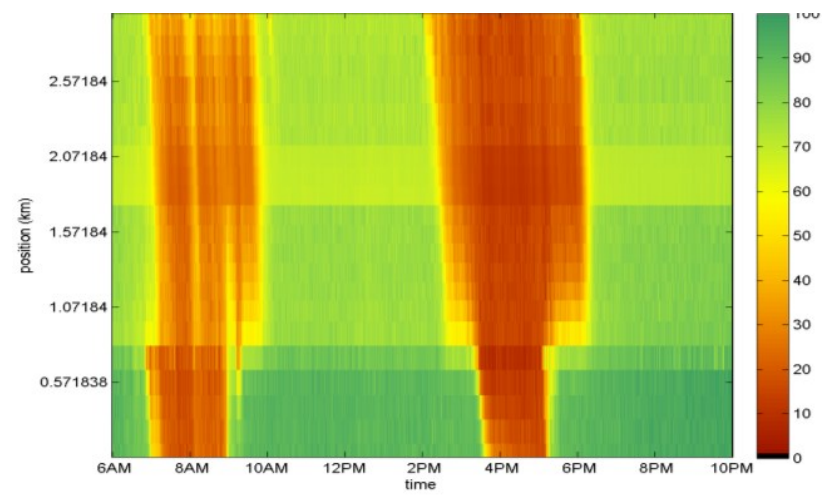

Figure 7. Space-time-speed plot for the model with two radar sensors (Scenario 3). The color-bar shows the speed in $\mathrm{km} / \mathrm{h}$.

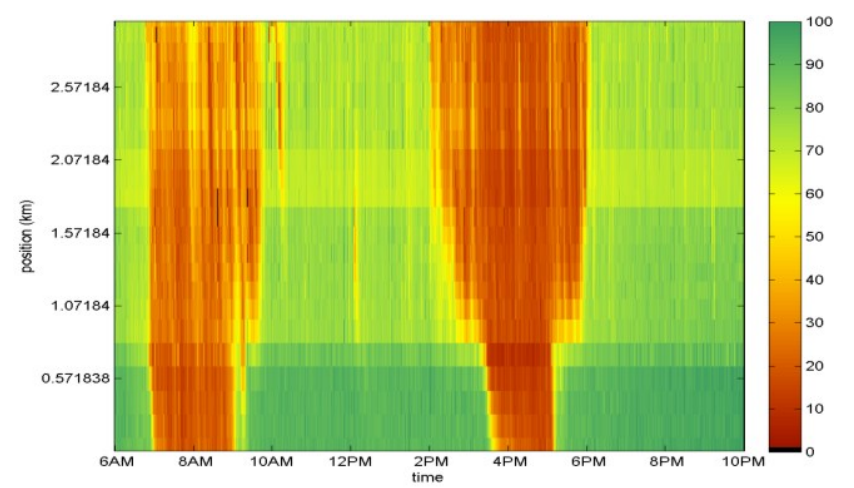

Figure 8. Space-time-speed plot for the model, two radar sensors and taxi (Scenario 4). The color-bar shows the speed in $\mathrm{km} / \mathrm{h}$. 


\section{DISCUSSION AND CONCLUSIONS}

This paper introduces a new method for fusing travel times with point speed measurements using a macroscopic traffic model and a non-linear filter. The system is tested using the CTM-v model together with the EnKF, but the method is possible to generalize for other models and filters as well. The method is efficient in handling spatial variations of the traffic state during the travel time observations and is most suitable when the spatial resolution of the estimation framework has higher resolution than the travel time observations. The method shows promising results in the evaluations on the test site using point speed observations from radars and travel time observations from taxis. However, the method works well also without point speed measurements, i.e. for data assimilation using CTM-V and only taxi probes. The method shows the best performance during build-up and discharge of the congestion, which is typically also the most challenging parts to estimate. During the congested state, the taxis seem to include a small bias compared to the radar and Bluetooth sensors, which could be caused by a different driver behaviour compared to regular drivers and an ability to do better lane choices than the average driver, which in a few cases causes very small improvements or even slightly worse results when adding the taxi observations. Considering this, the results could most likely be improved significantly by using dynamic covariance matrices for the different type of observations. It should also be noted that some of the bias could also be included in the radar measurements, which are known for underestimating the travel times during severe congestion.

The results in the paper are produced using the algorithm based on linearization of the measurement vector. Since the travel time as function of the cell speed is concave, the linearization of the travel time measurement is always an underestimate of the real travel time, which causes the filter to underestimate the cell speed vector. The results would most likely be improved by using the ensemble representation described in (16) and (17) instead, especially in the case where the predicted model state is far from the true state.

The method has a large potential in many systems for traffic estimation, since more heterogeneous sensors are deployed for many systems, incorporating in most cases both travel time observations as well as point speed observations. In the future many traffic estimation systems might rely fully on probe measurements, and this method can be of importance if one wants to fully utilize both the point speeds and the travel times reported in most recent probe systems.

Future work includes evaluation of the method using more data, using different performance metrics and comparing it with the naïve approach of fusing travel times and point speeds.

\section{REFERENCES}

[1] Gustafsson, F. (2010), Statistical Sensor Fusion, Studentlitteratur, Lund, Sweden.

[2] Hall, D.L. and Llinas, J. (1997), An Introduction to Multisensor Data Fusion, Proceedings of the IEEE, Vol. 85, pp. 6-23, Jan. 1997
[3] El Faouzi N-E., Leung, H. and Kurian, A. (2011), Data fusion in intelligent transportation systems: Progress and challenges - A survey, Information Fusion 12, 1 (January 2011), 4-10.

[4] Byon, Y.-J., Shalaby, A., Abdulhai, B. and El-Tantawy, S. (2010), Traffic Data Fusion using SCAAT Kalman Filters, In proceeding of the Transportation Research Board, 89th Annual Meeting, Washington, D.C.

[5] Bachmann, C., Abdulhai, B., Roorda, M. J. and Moshiri, B. (2013), A comparative assessment of multi-sensor data fusion techniques for freeway traffic speed estimation using microsimulation modeling, Transportation Research C: Emerging Technologies, vol. 26, pp. 33-48, 2013

[6] Bachmann, C., Roorda M. J., Abdulhai B. and Moshiri B. (2013), Fusing a Bluetooth Traffic Monitoring System with Loop Detector Data for Improved Freeway Traffic Speed Estimation, Journal of Intelligent Transportation Systems, Volume 17, Issue 2, 152-164, 2013

[7] Mitchell, H.B. (2007), Multi-sensor Data Fusion: An Introduction, Springer, NewYork, NY.

[8] Treiber, M. and Helbing, D. (2002), Reconstructing the spatio-temporal traffic dynamics from stationary detector data, Cooper@tive Tr@nsport@tion Dyn@mics 13.1-3.24.

[9] Van Lint, J.W.C. and Hoogendoorn, S. P. (2010), A Robust and Efficient Method for Fusing Heterogeneous Data from Traffic Sensors on Freeways. Computer-Aided Civil and Infrastructure Engineering, 25: 596-612.

[10] Van Lint J. W. C., (2010), Empirical evaluation of new robust travel time estimation algorithms, Transp. Res. Rec., vol. 2160, pp. 50-59.

[11] van Hinsbergen, C. P. I., Zuurbier, F. S., van Lint, J. W. C., and van Zuylen, H. J. (2008). Using an lwr model with a cell based extended kalman filter to estimate travel times. In Proceedings of the 3rd International Symposium of Transport Simulation. Surfer's Paradise, QLD, Australia

[12] Work, D., Blandin, S., Tossavainen, O.-P., Piccoli, B. and Bayen, A. (2010), A Traffic Model for Velocity Data Assimilation, Applied Mathematics Research eXpress (AMRX), April 2010(1), pp. 1-35,

[13] Mazare, P.-E., Tossavainen, O.-P., Bayen, A. and Work, D. (2012), Tradeoffs between inductive loops and GPS probe vehicles for travel time estimation: A Mobile Century case study, 91st Transportation Research Board Annual Meeting, Washington D.C., January 22-26, 2012.

[14] Hofleitner, A. and Bayen, A. (2011), Optimal decomposition of travel times measured by probe vehicles using a statistical traffic flow model, IEEE Conference on Intelligent Transportation Systems (ITSC), Washington, DC, pp. 815-821, October 2011.

[15] Qing, O., van Lint, J.W.C., Hoogendoorn, S.P. (2010), TravRes: A method for high-resolution traffic speed reconstruction using GPS-based travel-times, 13th International IEEE Conference on Intelligent Transportation Systems (ITSC), pp.1195-1201, 19-22 Sept. 2010

[16] M. Lighthill and G. Whitham. "On kinematic waves. II. A theory of traffic flow on long 742 crowded roads." Proceedings of the Royal Society of London. Series A, Mathematical and 743, Physical Sciences 229, no. 1178 (1955): 317-45.

[17] J. P. Lebacque. "The godunov scheme and what it means for first order traffic flow models." 734. In 13th International Symposium on Transportation and Traffic Theory, 647-77, 1996.

[18] A. Allstrom, J Archer, A. Bayen, S. Blandin, J. Butler, D. Gundlegard, H. Koutsopoulos, J. Lundgren, M. Rahmani, O.-P. Tossavainen, Mobile Millennium Stockholm, 2nd International Conference on Models and Technologies for ITS, Leuven, Belgium, June 22-24, 2011

[19] G. Evensen. Data Assimilation: The Ensemble Kalman Filter. SpringerVerlag, Berlin Heidelberg, 2007

[20] A. Allström, A. Bayen, M. Franssona, D. Gundlegård, A Patire, C. Rydergren, M. Sandin. Calibration Framework based on Bluetooth Sensors for Traffic State Estimation Using a Velocity based Cell Transmission Model. Transportation Research Procedia, Volume 3, 2014, Pages 972-981 